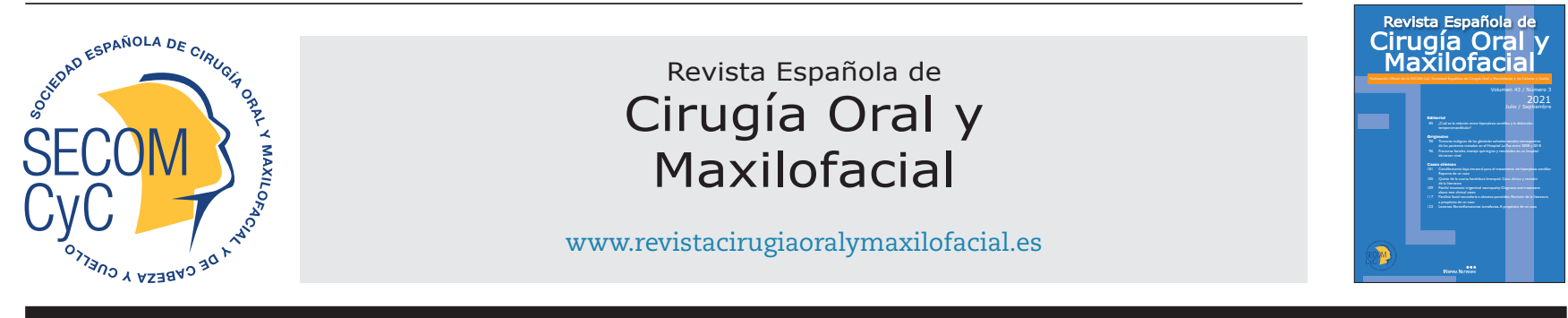

\title{
Original
}

\section{Tumores malignos de las glándulas salivales: estudio retrospectivo de los pacientes tratados en el Hospital La Paz entre 2008 y 2018}

\author{
Noelia Machío ${ }^{1}$, Juan José Pozo Kreilinger ${ }^{2}$, María Barajas Blancoํㅓㄴ José Luis del Castillo \\ Pardo de Vera ${ }^{1}$, Albert Malet Contreras ${ }^{3}$, Miguel Burgueño García ${ }^{1}$ y José Luis Cebrián \\ Carretero $^{1^{*}}$
}

${ }^{1}$ Servicio de Cirugía Oral y Maxilofacial, Hospital Universitario La Paz. Madrid, España. ${ }^{2}$ Servicio de Anatomía Patológica. Hospital Universitario La Paz. Madrid, España. ${ }^{3}$ Servicio de Cirugía Oral y Maxilofacial, Hospital San Juan de Dios. Barcelona, España

\section{INFORMACIÓN DEL ARTÍCULO}

Historia del artículo:

Recibido: 26 de mayo de 2021

Aceptado: 20 de septiembre de 2021

Palabras clave:

Glándulas salivales, cabeza y cuello, tumores malignos.

\section{R E S U M E N}

\begin{abstract}
Introducción: Los tumores malignos de glándulas salivares son neoplasias malignas poco frecuentes, con una mayor incidencia en la glándula parótida. A pesar de su baja incidencia, las posibles complicaciones derivadas de los mismos pueden conllevar gran comorbilidad en los pacientes.

Material y métodos: Hemos realizado un estudio retrospectivo, observacional y descriptivo de 105 pacientes con diagnóstico de neoplasias malignas de glándulas salivales en el Hospital Universitario La Paz. 102 corresponden a tumores primarios y 3 de ellos son metástasis de origen pulmonar.

Resultados: En nuestra serie la glándula parótida es el lugar de asiento más frecuente. Histológicamente, el carcinoma adenoide quístico es el tumor más común. El 75,23\% de los pacientes fueron diagnosticados con una citología mediante punción y aspiración con aguja fina (PAAF), y los restantes casos con una biopsia. El tratamiento más indicado fue la cirugía, sobre todo para aquellos tumores de estirpe epitelial. Un 33,5 \% recibió tratamiento adyuvante. Un $18 \%$ de los pacientes que sufrieron complicaciones postquirúrgicas presentó parálisis facial. Durante el desarrollo de la enfermedad, el 23,80 \% del total desarrolló metástasis a distancia, siendo el pulmón el órgano más afectado. El 26,66 \% de los pacientes sufrió al menos una recidiva. La supervivencia a los 2 años fue del 87,7 \% y a los 5 años del 75,5\%.

Discusión: A diferencia de otros estudios, en nuestro caso la estirpe más frecuente es el carcinoma adenoide quístico, lo cual podría explicarse porque se trata de un hospital de referencia para el tratamiento de la parálisis facial. Uno de los puntos más importantes en el manejo de estos pacientes es, no solo el control locorregional y a distancia, sino también el tratamiento de las posibles secuelas.
\end{abstract}

\footnotetext{
${ }^{*}$ Autor para correspondencia:

Correo electrónico: rodrigator2001@hotmail.com (José Luis Cebrián Carretero).

DOI: 10.20986/recom.2021.1291/2021

1130-0558/@ 2021 SECOM CyC. Publicado por Inspira Network. Este es un artículo Open Access bajo la licencia CC BY-NC-ND (http://creativecommons.org/licenses/by-nc-nd/4.0/).
} 


\section{Malignant salivary gland tumors: a retrospective study of patients treated at La Paz hospital between 2008 and 2018}

Keywords:

Salivary glands, head and neck, malignant tumors.

\begin{abstract}
A B S T R A C T
Introduction: Salivary gland tumors are rare malignant neoplasms, with a higher incidence in the parotid gland. Despite their low incidence, the possible complications derived from them can lead to high comorbidity in patients.

Methods: We conducted a retrospective, observational and descriptive study of 105 patients diagnosed with malignant neoplasms of the salivary glands at the Hospital Universitario La Paz. 102 correspond to primary tumors and 3 of them are metastases of pulmonary origin. Results: In our series the parotid gland is the most frequent site. Histologically, adenoid cystic carcinoma is the most common tumor. The $75.23 \%$ of the patients were diagnosed with cytology by fine needle aspiration and puncture (FNA), and the remaining cases with biopsy. The most indicated treatment was surgery, especially for tumors of epithelial lineage. Some $33.5 \%$ received adjuvant treatment. Some $18 \%$ of the patients who suffered post-surgical complications presented facial paralysis. During the course of the disease, $23.80 \%$ of the total developed distant metastases, the lung being the most affected organ. Of the patients, $26.66 \%$ suffered at least one recurrence. Survival at 2 years was $87.6 \%$ and at 5 years $75.3 \%$. Discussion: Unlike other studies, in our case the most frequent lineage of salivary glands tumors is adenoid cystic carcinoma. This could be explained because our hospital is a reference in facial paralysis. One of the most important points in the management of these patients is not only the local and distant control of the tumor, but also the treatment of possible sequelae.
\end{abstract}

\section{INTRODUCCIÓN}

Los tumores malignos de glándula salival constituyen el 0,3 \% de todas las neoplasias malignas, y entre el 1-3\% de las neoplasias de cabeza y cuello. Alrededor del $75 \%$ de los tumores son benignos. La parótida es el lugar donde más frecuentemente asientan estas neoplasias, siendo las glándulas salivales menores la segunda localización más frecuente (sobre todo en el paladar), siendo la gran mayoría de ellas malignas (70-75\%). A la glándula submaxilar le corresponden un $8 \%$ de los tumores; de ellos, el 40-45 \% son malignos. Por último, la glándula sublingual es la que con menor frecuencia desarrolla tumoraciones, únicamente un $1 \%$, aunque el 70-90 \% de ellas son malignas ${ }^{1,2}$.

La etiología de estos tumores es desconocida debido a que no hay un factor de riesgo predominante que pueda ser asociado al desarrollo del mismo. No obstante, hay una serie de factores que se han propuesto como causas potenciales.

Clínicamente se caracterizan como masas de crecimiento rápido y consistencia pétrea. Además, la presencia de parálisis facial debe hacernos sospechar la presencia de malignidad. Asimismo, el dolor es indicativo de afectación nerviosa sensitiva y, por tanto, implica un peor pronóstico y reducción de la supervivencia de hasta un $35 \%{ }^{1}$. Por otro lado, los tumores de las glándulas salivales menores pueden presentarse bien como una masa submucosa indolora, o bien como ulceración de la mucosa, ya sea en el paladar, los labios o la mucosa bucal. Los síntomas ocasionados por este tipo de tumores en estadios más avanzados dependen de la ubicación del tumor y pueden incluir obstrucción nasal, congestión, cambios en la visión o trismo cuando están presentes en la cavidad nasal o el seno maxilar. Cuando afectan a la nasofaringe, suelen presentarse en un estadio avanzado y es frecuente la invasión de la base del cráneo, la extensión intracraneal o la afectación de los nervios craneales ${ }^{3}$.

El diagnóstico histológico es necesario para confirmar la sospecha de neoplasia maligna. Actualmente, el estudio citológico mediante la punción y aspiración con aguja fina (PAAF) en manos expertas alcanza una especificidad del $98 \%$ y una sensibilidad del $80 \%$, y resulta muy útil para clasificar una neoplasia como benigna o maligna e identificar el subtipo tumoral. La biopsia incisional suele reservarse para el diagnóstico de tumores en glándulas salivales menores ${ }^{4,5}$.

Respecto a las pruebas de imagen, la resonancia magnética (RM) puede ser necesaria para evaluar la extensión, la invasión local, ósea y perineural y las metástasis de los ganglios linfáticos, así como para evaluar el espacio parafaríngeo ${ }^{5}$.

El manejo terapéutico de estos pacientes tiene como objetivo fundamental controlar la enfermedad y conservar la función del nervio facial cuando sea posible ${ }^{4}$. La resección quirúrgica completa es la opción de elección cuando se pueden lograr márgenes quirúrgicos libres de infiltración. Los pacientes con neoplasias malignas de bajo grado generalmente se suelen tratar únicamente con cirugía. Los pacientes con carcinomas de alto grado suelen requerir cirugía y radioterapia (RT) adyuvante, con o sin quimioterapia (QT) o inmunoterapia asociada.

Se debe tomar la decisión de sacrificar el nervio facial cuando esté afectado de forma preoperatoria si compromete la resección de un tumor maligno, o en el caso de que el paciente presente una parálisis facial previa, lo que indicaría que el nervio está afectado. En este caso, la cirugía realizada se denomina parotidectomía radical. La parálisis facial pre- 
operatoria está presente en el 15-23 \% de los pacientes con tumor maligno de glándula parótida y suele implicar un peor pronóstico. Aproximadamente el 20 \% de los pacientes con tumores malignos de las glándulas salivales tienen metástasis ganglionares clínicamente aparentes en el momento diagnóstico, y deben ser tratados con vaciamiento cervical seguido de RT adyuvante en la mayoría de los casos ${ }^{6}$.

La RT adyuvante se considera una buena opción en pacientes con factores de mal pronóstico tras la cirugía, como tumores de alto grado, estadios avanzados, márgenes quirúrgicos positivos, invasión cutánea, nerviosa o linfática, así como prácticamente para todos los carcinomas adenoides quísticos ${ }^{1}$.

En cuanto a la inmunoterapia, hasta el $30 \%$ de los carcinomas mucoepidermoides y el $40 \%$ de carcinomas ductales sobrexpresan el receptor del factor de crecimiento epidérmico humano 2 (HER2) o tienen amplificación de ERRB2 (el protoncogen de HER2). En estos casos ofrecemos quimioterapia basada en taxanos en combinación con trastuzumab como tratamiento de primera línea ${ }^{7}$.

La sobreexpresión del EGFR en los carcinomas tiende a predecir una mayor incidencia de metástasis cervicales y recurrencias ${ }^{8}$, por lo que hay estudios que plantean la posibilidad de emplear cetuximab ${ }^{9}$.

El objetivo del presente estudio es analizar la casuística del Hospital Universitario La Paz durante el periodo entre el 2008 y 2018 para definir las características epidemiológicas de los distintos tipos histológicos de los tumores malignos salivales, así como su tratamiento y evolución.

\section{MATERIAL Y MÉTODOS}

Se diseña un estudio observacional, descriptivo, retrospectivo y monocéntrico sobre los tumores malignos de glándulas salivales diagnosticados e intervenidos entre el 2008 y el 2018.

Analizamos las historias clínicas de los pacientes desde una base de datos elaborada a partir de la herramienta HCIS y el Programa PATWIN de Anatomía Patológica. Se recogieron datos de 105 pacientes y se estructuraron en una tabla Excel para el posterior análisis estadístico a partir de las variables a estudiar. Estas fueron: edad, sexo, localización del tumor, tipo histológico, tratamiento, método diagnóstico, complicaciones, desarrollo de enfermedad a distancia, recidivas, supervivencia a los dos años y a los cinco años.

Los datos fueron anonimizados en todo momento, ya que a cada historia se le asignó un número desde el 1 en adelante, comenzando por el primer año de estudio.

Para el análisis de datos se utilizó el programa SPSS y los cálculos que se utilizaron fueron correspondientes a estadís- tica descriptiva con frecuencias, así como tablas de concentración de resultados y gráficas descriptivas.

\section{RESULTADOS}

Con los criterios diagnósticos de inclusión se obtuvieron 105 casos. 102 fueron tumores primarios de las glándulas salivales y los 3 restantes se clasificaron como tumores secundarios (metástasis en glándulas salivales de tumores primarios de otras localizaciones). Del total de pacientes, 59 eran mujeres y 46 eran hombres.

La edad media de diagnóstico en nuestra serie fue 56,3 años. Según el sexo fue 55,5 años para los hombres y 57 años para las mujeres. La mediana fue 60 años para los hombres y 59 para las mujeres (Tabla I).

La glándula parótida fue la localización más frecuente (47,6 \%), seguida de las glándulas salivales menores (36,1\%), la glándula submaxilar (15,2 \%) y la glándula sublingual (0,9\%).

El tumor más frecuente a nivel global fue el carcinoma adenoide quístico (22,9\%), seguido por el carcinoma mucoepidermoide (20,9\%). De estos últimos, la mayoría eran de bajo grado (61,9 \% del total de carcinomas mucoepidermoides diagnosticados) (Tabla II).

También se analizó qué tipo de tumor es más frecuente para cada glándula. Según nuestro estudio, el tumor más frecuente en la glándula parótida es el carcinoma de células acinares, seguido del carcinoma mucoepidermoide y a continuación del carcinoma adenoide quístico.

En la glándula submaxilar, el tipo histológico más frecuente es el carcinoma adenoide quístico. En las glándulas salivales menores, el más frecuente es el carcinoma mucoepidermoide (Tabla III).

En cuanto a la clínica, la mayoría de los pacientes refieren la palpación de una masa cervicofacial como forma de debut y motivo de consulta (82,4\%). La parálisis facial estaba presente en el 6,6 \% de los casos.

De los 105 pacientes, a 79 de ellos (75,23\% del total) se le realizó una citología previa al tratamiento para filiar el tipo histológico. Al 53 \% se realizó en la glándula parótida. Los 26 restantes tienen registrada una biopsia realizada en nuestro centro, y la mayoría de ellas para diagnosticar tumores de glándulas salivales menores.

Respecto al tratamiento, 104 pacientes fueron tratados y únicamente un paciente no lo fue debido a su situación basal.

El tratamiento de elección en los tumores parotídeos fue la parotidectomía total o radical con vaciamiento cervical (el $43 \%$ del total de los pacientes con tumores parotídeos). En la glándula submaxilar, el tratamiento de elección en todos

Tabla I. Media, mediana y moda de la variable edad de diagnóstico según el sexo.

\begin{tabular}{clcccccc}
\multicolumn{10}{c}{ Edad DX } \\
\hline & & Media & Mediana & Moda & Mínimo & Máximo & Desviación estándar \\
\hline \multirow{3}{*}{ Sexo } & Hombre & 55,5 & 60,0 & 60,0 & 12,0 & 89,0 & 19,1 \\
& Mujer & 57,0 & 59,0 & 49,0 & 13,0 & 87,0 & 17,3 \\
& Total & 56,3 & 60,0 & 63,0 & 12,0 & 89,0 & 18,0
\end{tabular}


Tabla II. Tipo histológico tumoral más frecuente en total.

\begin{tabular}{lcc} 
& Frecuencia & Porcentaje \\
\hline CA adenoide quístico & 24 & 22,9 \\
CA mucoepidermoide & 22 & 20,9 \\
CA células acinares & 16 & 15,2 \\
CA ductal & 11 & 10,5 \\
Linfoma no Hodgkin B tipo Malt & 6 & 5,7 \\
ADC polimorfo & 5 & 4,7 \\
CA epimioepitelial & 3 & 2,8 \\
CA linfoepitelial & 3 & 2,8 \\
ADC & 2 & 1,9 \\
ADC acinar variante oncocitica & 1 & 0,9 \\
CA células basales & 1 & 0,9 \\
CA células claras & 1 & 0,9 \\
CA epidermoide & 1 & 0,9 \\
CA indiferenciado de célula & 1 & 0,9 \\
grande & & \\
CA microcítico/célula pequeña & 1 & 0,9 \\
CA mioepitelial & 1 & 0,9 \\
CA neuroendocrino & 1 & 0,9 \\
Cistoadenocarcinoma papilar & 1 & 0,9 \\
Linfoma del manto & 1 & 0,9 \\
ADC pulmón & 3 & 2,8 \\
Total & 105 & 100,0 \\
\hline CA carcinom ADC: & &
\end{tabular}

CA: carcinoma. ADC: adenocarcinoma. los casos fue la submaxilectomía. En las glándulas salivales menores, la técnica más empleada fue la tumorectomía con márgenes (el 58 \% de los pacientes con tumores en glándulas salivales menores).

El 33,5 \% del global de los pacientes recibieron tratamiento adyuvante con quimioterapia, radioterapia o ambas.

Respecto a las complicaciones o secuelas, el 55,66 \% de los pacientes sufrió alguna complicación. De ellas, la más incapacitante fue la parálisis facial postquirúrgica (19 pacientes; $18 \%$ del total), y síndrome de Frey (2 pacientes; 1,9\% del total).

Durante el seguimiento de la enfermedad, un 5,6 \% de los pacientes presentó afectación linfática y fue tratado quirúrgicamente con disección cervical.

Considerando la diseminación metastásica, 25 pacientes (23,8\% del total) han presentado metástasis en otras localizaciones. Dentro de este grupo, el $42 \%$ se produjo en el pulmón, el $30 \%$ en el hueso, el $21 \%$ afectó al sistema nervioso central, el $3 \%$ en páncreas y el $3 \%$ en hígado.

El carcinoma adenoide quístico fue el origen más común de metástasis pulmonares.

28 pacientes de los 105 incluidos en el estudio sufrieron alguna recidiva local (26,6 \% del total). El tipo histológico que más recidivas presentó fue el adenoide quístico, con un total de 11 recidivas, seguido del carcinoma ductal y adenocarcinoma, presentando 5 recidivas cada uno.

En lo relativo a la supervivencia, se ha comprobado que a los 2 años es del 87,6 \%, y a los 5 años del 75,3\%.

\section{Tabla III. Tipo histológico tumoral más frecuente según la glándula afecta.}

\begin{tabular}{|c|c|c|c|c|c|}
\hline Tipo de tumor & Glándula salivar menor & Parótida & Sublingual & Submaxilar & Total porcentual \\
\hline CA adenoide quístico & $7,63 \%$ & $8,58 \%$ & - & $6,68 \%$ & $22,89 \%$ \\
\hline CA células acinares & $1,90 \%$ & $11,40 \%$ & - & $1,90 \%$ & $15,2 \%$ \\
\hline CA mucoepidermoide & $10,48 \%$ & $9,52 \%$ & - & $0,95 \%$ & $20,95 \%$ \\
\hline CA ductal & $2,86 \%$ & $5,73 \%$ & - & $1,91 \%$ & $10,5 \%$ \\
\hline Linfoma no Hodgkin B tipo Malt & $2,85 \%$ & $2,85 \%$ & - & - & $5,7 \%$ \\
\hline ADC polimorfo & $4,7 \%$ & - & - & - & $4,7 \%$ \\
\hline CA epimioepitelial & - & $1,9 \%$ & - & $0,95 \%$ & $2,85 \%$ \\
\hline CA linfoepitelial & - & $2,8 \%$ & - & - & $2,8 \%$ \\
\hline ADC & $0,95 \%$ & $0,95 \%$ & - & - & $1,9 \%$ \\
\hline ADC acinar variante oncocitica & - & - & - & $0,95 \%$ & $0,95 \%$ \\
\hline CA células basales & $0,95 \%$ & - & - & - & $0,95 \%$ \\
\hline CA células claras & - & - & $0,95 \%$ & - & $0,95 \%$ \\
\hline CA epidermoide & - & $0,95 \%$ & - & - & $0,95 \%$ \\
\hline CA indiferenciado de célula grande & - & $0,95 \%$ & - & - & $0,95 \%$ \\
\hline CA microcítico/célula pequeña & - & - & - & $0,95 \%$ & $0,95 \%$ \\
\hline CA mioepitelial & $0,95 \%$ & - & - & - & $0,95 \%$ \\
\hline CA neuroendocrino & - & $0,95 \%$ & - & - & $0,95 \%$ \\
\hline Cistoadenocarcinoma papilar & $0,95 \%$ & - & - & - & $0,95 \%$ \\
\hline Linfoma del manto & $0,95 \%$ & - & - & - & $0,95 \%$ \\
\hline ADC pulmón & $0,95 \%$ & $0,95 \%$ & - & $0,95 \%$ & $2,85 \%$ \\
\hline Total & $36,12 \%$ & $47,53 \%$ & $0,9 \%$ & $15,24 \%$ & $100,0 \%$ \\
\hline
\end{tabular}

CA: carcinoma. ADC: adenocarcinoma. 
Por último, cabe destacar que en el grupo minoritario correspondiente a tumores malignos de glándulas salivales metastásicos, el origen primario en los tres casos fue un adenocarcinoma de origen pulmonar. Los tumores se desarrollaron uno en glándulas salivales menores, otro en la glándula parótida y el restante en la glándula submaxilar.

\section{DISCUSIÓN}

Los tumores malignos de glándulas salivales constituye el $0,3 \%$ de todas las neoplasias malignas. En nuestro estudio se aprecia que la glándula parótida es el lugar de asiento preferente para las neoplasias malignas (47,6\%), debido a que la incidencia de tumoraciones en esta glándula es superior al resto.

Actualmente estos tumores malignos son más frecuentes en mujeres, lo cual también se evidencia en nuestra serie, sobre todo en la glándula parótida, donde el 67 \% eran mujeres.

En cuanto a la edad, en nuestra muestra la edad media de diagnóstico en mujeres es de 57 años (mediana de 59), y en hombres es de 55,5 años (mediana de 60). En total, la edad media de diagnóstico es de 56,3 y mediana de 60. Esta diferencia entre la media y la mediana se debe a que la menor edad es de 12 años y la máxima es de 89 años, lo que implica que el rango es de 77 años ${ }^{2}$.

El tumor más frecuente en nuestro estudio fue el carcinoma adenoide quístico. Esto no concuerda con los datos reflejados en la bibliografía internacional, donde figura el carcinoma mucoepidermoide. Se justificaría teniendo en cuenta que el Hospital Universitario La Paz es un centro de referencia en parálisis facial. El carcinoma adenoide quístico se suele presentar como una masa sólida de crecimiento lento con tendencia a la invasión perineural y extraparenquimatosa ${ }^{8}$. Esto hace que la frecuencia de afectación del nervio facial sea elevada.

El carcinoma mucoepidermoide en nuestra serie es el segundo en frecuencia global. La mayoría de ellos fueron diagnosticados de bajo grado. En nuestra serie fue el tumor más frecuente en las glándulas salivales menores, al contrario de lo que ocurre en la mayor parte de las series publicadas, donde se propone al carcinoma adenoide quístico como el más frecuente ${ }^{10}$.

Respecto al diagnóstico, hoy en día es imprescindible contar con un estudio citológico a través de la PAAF para identificar el tipo de células y plantear el tratamiento adecuado. De los 105 pacientes, a 79 de ellos (75,23 \% del total) se les realizó una citología previa al tratamiento para filiar el tipo histológico. Los 26 restantes son pacientes con tumores en las glándulas salivales menores y se les realizó una biopsia incisional.

Actualmente la RMN multiparamétrica es una técnica útil y eficaz en el estudio de los tumores de glándulas salivales. Las secuencias morfológicas permiten definir el grado de extensión local de la lesión, así como su localización-extensión exacta, hechos absolutamente relevantes en la consideración de su tratamiento ${ }^{11}$.

En nuestra serie de pacientes, la mayoría fueron tratados de entrada con un tratamiento quirúrgico, sobre todo aquellos tumores de extirpe epitelial, realizando una extirpación con márgenes libres.

El tratamiento de entrada con quimio y/o radioterapia se reservó para los casos de linfoma no Hodgkin tipo B MALT y linfoma del Manto. Como tratamiento adyuvante se empleó para el 33,5\% pacientes de la serie.

Respecto a las complicaciones y secuelas, la más limitante es la parálisis facial postquirúrgica. El 18 \% de nuestros pacientes sufrieron esta complicación. Esto se ajusta con lo hallado en la literatura, donde en los tumores malignos de la glándula parótida la incidencia de parálisis del nervio facial está presente en un 27-43\% de manera temporal y en un 4-22 \% de manera permanente ${ }^{6}$.

En la evolución de nuestros pacientes, el 5,6 \% desarrolló una diseminación locorregional linfática y fue tratado con tratamiento quirúrgico y vaciamiento cervical. 25 pacientes (23,85 \% del total) han presentado metástasis, siendo el pulmón el órgano más afectado por las mismas, seguido del tejido óseo y a nivel del sistema nervioso central. Más de la mitad de los pacientes con afectación metastásica pulmonar tenían origen un carcinoma adenoide quístico. Su tasa de metástasis a distancia varía entre $25-50 \%$, según la literatura y la mayoría son pulmonares ${ }^{12}$.

La incidencia de recidivas en nuestra serie de pacientes es de $26,6 \%$. En los tumores de glándula submaxilar, la recidiva es menos frecuente porque la cirugía incluye la extirpación del tumor, tejido glandular sano que lo rodea, y la cápsula glandular. De estas, el tipo histológico que más recidivas presentó fue el adenoide quístico, con un total de 11 recidivas. Las recurrencias del carcinoma adenoide quístico son un caso común debido a su tendencia por la diseminación neural, vascular y ósea, y muchas de ellas se producen a los 10-20 años del tratamiento ${ }^{1}$.

En lo relativo a la supervivencia, en nuestro estudio se ha comprobado que a los 2 años es del 87,6 \%, y a los 5 años del $75,3 \%$. Esto es similar a los hallazgos en otros estudios donde la supervivencia a los 5 años está entre el 73-80\%.

\section{CONCLUSIONES}

A pesar de que los tumores malignos de glándula salivar son poco frecuentes, las secuelas derivadas de los mismos son potencialmente graves, sobre todo en el caso de la parálisis facial, que desarrollaron el $18 \%$ de nuestros pacientes. Para evitar dichas secuelas, es imprescindible el manejo multidisciplinar del paciente e intentar realizar rehabilitación quirúrgica de la forma más precoz posible. La localización más frecuente de los tumores malignos es la glándula parótida y el tipo de tumor maligno más común es el carcinoma adenoide quístico. Además, es importante realizar un correcto seguimiento de los pacientes, tanto clínico como radiológico, ya que durante la evolución de la enfermedad el 23,80 \% de los pacientes desarrollaron una enfermedad metastásica principalmente en pulmón.

\section{B I B L I O G R A F Í A}

1. Plaza G, Aparicio JM. Patología de las glándulas salivales [ponencia]. AMORL; 2016. Disponible en: http://www.amorl.es/ index.php/2016-07-08-09-57-26/ponencias-congresos/208ponencia-xi-congreso-amorl-patologia-de-las-glandulas-salivales-h-fuenlabrada-2016. 
2. To VSH, Chan JYW, Tsang RKY, Wei WI. Review of Salivary Gland Neoplasms. ISRN Otolaryngol. 2012;2012:872982.

3. Laurie SA. Salivary gland tumors: Epidemiology, diagnosis, evaluation, and staging. UpToDate; 2021 [Actualización febrero 2020]. Disponible en: https://www.uptodate.com/contents/ salivary-gland-tumors-epidemiology-diagnosis-evaluationand-staging.

4. Rey Biel J, Sánchez Aniceto GS, Salmerón Escobar JI, Martorell Martínez V. Tumores de la glándula parótida. En: Protocolos de la Sociedad Española de Cirugía Oral y Maxilofacial. Madrid: Sociedad Española de Cirugía Oral y Maxilofacial; 2014.

5. Schmidt RL, Hall BJ, Wilson AR, Layfield LJ. A systematic review and meta-analysis of the diagnostic accuracy of fine-needle aspiration cytology for parotid gland lesions. Am J Clin Pathol. 2011;136(1):45-59. DOI: 10.1309/AJCPOIEOCZNAT6SQ.

6. Lydiatt WM, Quivey JM. Salivary gland tumors: Treatment of locoregional disease. UpToDate; 2021 [Actualización marzo 2021]. Disponible en: https://www.uptodate.com/contents/ salivary-gland-tumors-treatment-of-locoregional-disease.

7. Laurie SA, Schiff B. Malignant salivary gland tumors: Treatment of recurrent and metastatic disease. UpToDate; 2021
[Actualización noviembre 2020]. Disponible en: https://www. uptodate.com/contents/malignant-salivary-gland-tumorstreatment-of-recurrent-and-metastatic-disease.

8. Young A, Okuyemi OT. Malignant Salivary Gland Tumors. 2020 Oct 13. In: StatPearls [Internet]. Treasure Island (FL): StatPearls Publishing; 2021.

9. Locati LD, Bossi P, Perrone F, Potepan P, Crippa F, Mariani L, et al. Cetuximab in recurrent and/or metastatic salivary gland carcinomas: A phase II study. Oral Oncol. 2009;45(7):574-8. DOI: 10.1016/j.oraloncology.2008.07.010.

10. Burke CJ, Thomas RH, Howlett D. Imaging the major salivary glands. Br J Oral Maxillofaci Surg. 2011;49(4):261-9. DOI: 10.1016/j.bjoms.2010.03.002.

11. Brea Álvarez B. RM multiparamétrica en el estudio de los tumores de las glándulas salivales [Tesis doctoral]. Madrid; Universidad Autónoma de Madrid; 2015. Disponible en: https:// repositorio.uam.es/handle/10486/668012.

12. Spiro RH, Huvos AG, Strong EW. Adenoid cystic carcinoma of salivary origin. A clinicopathologic study of 242 cases. Am J Surg. 1974;128(4):512-20. DOI: 10.1016/0002-9610(74) 90265-7. 